\title{
Quality Indicator Measures as It Affects Turnaround Time (TAT) in A Molecular Laboratory in Port Harcourt, Rivers State
}

\author{
B. W. Moore-Igwe, S. G. Christian, R.B. Jacob, T. Odinga, and E.M. Eze
}

\section{ABSTRACT}

Background: Turnaround Time (TAT) is an important Quality Indicator in the medical laboratory. The Rivers State University Teaching Hospital (RSUTH) Polymerase Chain Reaction (PCR) laboratory was enrolled in the process of World Health Organisation (WHO) - Regional Office for Africa (AFRO) accreditation by FHi360 in preparation for the ISO 15189 accreditation in 2016. One of the services rendered in the laboratory is Early Infant Diagnosis (EID)/Dried Blood Spots (DBS) in Human Immunodeficiency Virus (HIV) exposed infants. Clinicians depend on these results to determine the next step for the management of HIV exposed Infants. This study is aimed at assessing the rate of sample rejection $(\mathrm{SR})$, determine the effect of specific intervention on this rate and the effect of SR on TAT.

Method: It involves the assessment of samples delivered to the RSUTH PCR Laboratory from January 2019 to March 2020. A baseline rate of sample rejection was established from January to July 2019. Interventional measures were put in place such as introducing the national algorithm for rejection and acceptance of samples, training was also done for EID sample collectors and a final assessment of changes in the rate of sample rejection was determined at the final period of January to March 2020.

Results: During the baseline period, sample rejection rate started at $5 \%$ in February and went back to $0 \%$ in March. In April however, the rate of rejection increased to $9 \%$. There was a decline in rejection rate to $5 \%$ and $7 \%$ in May and June respectively. A sudden spike in rejection occurred in July at a rate of $19 \%$. The major reasons for sample rejection were DBS cards with insufficient blood spots, DBS cards with clots present in spots, DBS cards that have serum rings and grossly haemolysed DBS. After baseline data was collected and interventions put in place. Sample rejection rate drastically reduced to $1 \%, 0 \%$ and $0 \%$ respectively from January to March which is way below the maximum threshold of $2 \%$ as advocated by WHO. At baseline EID, TAT was longer than a month, however; with SR, the TAT increased to about seven weeks. The final assessment in March from this study showed a significant reduction in sample rejection to $0 \%$.

Conclusion and recommendations: This study has shown that quality improvement is achievable if interventional tools are utilized promptly. This will result in shorter TAT; fewer samples rejected and therefore prompt treatment of exposed infants thus reducing morbidity and mortality due to HIV.

Keywords: Dried blood spot, early infant diagnosis, HIV, sample rejection, turn around time.

\section{INTRODUCTION}

Turnaround Time (TAT) involves three different phases; pre-analytical phase (sample ordering, sample collection, sample identification, sample transport, sample reception (where sample rejection often occurs); analytical phase (sample preparation and sample analysis) and the postanalytical phase (result reporting, result interpretation, and
Published Online: August 16, 2021

ISSN: $2736-5476$

DOI: 10.24018 /ejclinicmed.2021.2.4.109

B. W. Moore-Igwe*

Department of Medical Laboratory Science Rivers State University Port Harcourt, Rivers State, Nigeria

(e-mail: beatrice.moore-igwe1@ust.edu.ng) S. G. Christian

Department of Medical Laboratory Science Rivers State University Port Harcourt, Rivers State, Nigeria.

(e-mail: serekara.christian1@ust.edu.ng)

R. B. Jacob

Department of Medical Laboratory Science Rivers State University Port Harcourt, Rivers State, Nigeria.

(e-mail: ransom.jacob@ust.edu.ng)

T. Odinga

Department of Biochemistry Rivers State University Port Harcourt, Rivers State, Nigeria.

(e-mail: bomaodinga@gmail.com)

E. M. Eze

Department of Medical Laboratory Science Rivers State University Port Harcourt, Rivers State, Nigeria.

(e-mail: evelyn.eze@ust.edu.ng)

*Corresponding Author action by the clinician). Hence TAT simply put refers to the time when requisition of test is made, to when clinical decision is made [1].

When a client derives satisfaction from a product or a service, quality is said to have been achieved [2]. Most laboratories avoid talking about quality but rather look into issues of imprecision and inaccuracy. Contrary to this, however, clinicians would rather have quality service, which 
includes imprecision and inaccuracy, availability, cost effectiveness, priority, and time bound [3]. Time bound among others seem to take first place [3]. Turnaround time indeed is a pivotal quality indicator of how effective a laboratory performs [4]. Hence, prolonged TAT gives room for clients to complain and even query the quality of the tests [5]. Turnaround time if not tackled, creates room for constant complaint from one client to the other and is faced with issues of conflict resolution which will affect the duty of the conflict resolver(s). Very many laboratories still prefer to go the conventional way rather than imbibe the recent technological changes, this hampers on TAT. Constant retrieval and evaluation of data should be part of a laboratory quality management to recognize, properly handle, always troubleshoot, and develop a better performance plan and safety of patient by recognizing and carrying out valuable interventional measures with the intension of robust standards of vital key practices in laboratories [6], [7].

Early Infant Diagnosis (EID) or Dried Blood Spot (DBS) is a qualitative test for babies born to HIV mothers who may still have maternal antibody in circulation hence may test false positive to rapid test kits but have their samples tested using PCR within 6 weeks to 18 months after birth.

Global epidemiology of HIV in infants is deduced from their mothers at an estimation of 1.4 million in 2008 since acquisition of HIV in infancy is almost all from mother to child in developing nations with the occurrence of 430,000 new pediatric cases [8]. Approximation of $15 \%-30 \%$ occurs, when ART is not given to these infants exposed to HIV mothers at the time of pregnancy. Childbirth and long-term breast feeding of these infants can also result in $10 \%-20 \%$ risk of transmission [9]. All this could be due to prolonged TAT, sample rejection, equipment down time and reagent stock out.

Progression of HIV occurs rapidly in exposed infants with increased mortality if commencement of ART is delayed and appropriate care is not given, one-third of infants die in their first year of life and almost half of such infants do not make it to their second year of their life. Infants who seem to be healthy carriers at less than 18 months do not undergo ART, and usually lack appropriate care often [10]. Commencement of ART as well as appropriate care can only be enabled if diagnosis is detected early enough as advocated by World Health Organization (WHO), which impacts positively as death and long term stay in the hospital is ruled out [8].

HIV antibody tests, including newer tests, do not establish the presence of HIV infection in infants because of transplacental transfer of maternal immunoglobulin to HIV; therefore, a virologic test should be used [11]. Varying options can be used to establishing HIV in infants below 18 months. Among which is the polymerase chain reaction (DNA PCR), for detection of HIV-1 pro-viral DNA which incorporates in the genome of human [12],[14]. New outbreaks of HIV in exposed infants through pregnancy, birth canal and even by breast feeding must be stopped in order to achieve the fast-track targets of ending the AIDS epidemic by 2030. Early commencement of ART can also reduce transmission rates to $5 \%$ or less [15]. Achieving accurate diagnosis is only enabled when samples are of optimal quality and processed as soon as they get to the laboratory [11].

The Prevention of Mother-to-Child Transmission of HIV
(PMTCT) program has added value to the efficiency and wide reach of interventions which has resulted in the huge number of exposed infants born without the virus [16]. Nevertheless, early detection of infants before disease strikes can be achieved through routine diagnostic checks of all exposed infants, prioritizing TAT, since prolonged TAT may result in mortality. Hence WHO advocates that two virologic tests be carried out at 6 weeks of age and immediate placement of lifelong ART [17].

In Nigeria, there is an increased access to ART at varying levels: tertiary, secondary and primary health care facilities. Recognition of identified infants is fast tracked due to rapid disease progression in these infants [18]. Collection of samples for the DNA-PCR from exposed infants occur in the form of a dried blood spot (DBS) which is properly packaged and sent to the Hub for analysis. The sample is sent alongside a sample requisition form which incorporates patient unique identifiers (such as hospital number, district/Local Government Area (LGA) code, sending, mother's name and ART information, infant's name, sex, date of birth and information on breastfeeding). It also captures the date the sample was taken, mode of delivery, PMTCT prophylaxis, and infant's feeding practice, reasons for the DNA-PCR test, entry point for the test, and finally the result of the test [19].

When quality of EID samples become suboptimal, it affects the number of samples accepted, hence rejection rate will enormously increase with an overall negative impact on TAT. A study carried out in the National Microbiology Reference Laboratory (NMRL) showed a total of 2020 (3.5\%) samples were rejected, this affected TAT as results were rather delayed [20]. So many reasons were responsible for rejecting the samples, predominantly insufficient samples, sample contamination, and disparity between sample and form, these do not synchronize with the Standard Operating Procedure (SOP) for collection, storage, and transportation of DBS samples [21].

Rejection of samples leads to delay in providing critical lifelong antiretroviral therapy to deserving children and is contributory to the prevailing $43 \%$ pediatric ART initiations, against $74 \%$ for adults in Zimbabwe [19]. The rise in the rejection rates, above the World Health Organization (WHO) maximum threshold of $2 \%$, presents an urgent need to evaluate the existing EID sample management mechanisms and the extent of adherence to them in order to enhance pediatric ART initiations [21].

The laboratory medicine is the focal point of the health care system. Patient care wholly depends on laboratory information. This emphasizes the call for excellent time bound results.

\section{Methodology}

The project was conducted in the PCR Laboratory of Rivers State University Teaching Hospital (RSUTH), Port Harcourt from January 2019 to March 2020. This PCR laboratory is a Hub which analyses EID samples from over 250 facilities that render ART service in Rivers State and some neighboring States. Records of samples rejected were collected from the sample rejection $\log$ at the reception; number of rejected samples within seven months were counted. Microsoft Excel 2010 program was used for Statistical analysis. Presentation of calculated rejected 
samples was presented in percentages and as numbers. Reasons for EID rejection which consequently impacted on TAT were the following: DBS cards with insufficient blood spots (need to have at least three good spots), DBS cards with clots present in spots, DBS cards that have serum rings indicating contamination around the spots, grossly hemolyzed. Others were: Poorly labeled/unlabeled sample, mismatched sample, missing labels on package or tracing form, form with no sample/sample with no form, inappropriate sample packing. Reasons for suboptimal preparation of EID samples were Staff incompetency (staff who prepares DBS spot), improper step - down training for: newly posted staff, new employees, and old staff, sudden relieve of duty from those already trained, criteria for sample acceptance /rejection not put in place.

After the baseline rejection analysis rate was done, step down trainings were conducted in collaboration with the implementing partners FHi36o in various facilities with poor competent staff, distribution of algorithm for acceptance and rejection of EID samples, facility heads were encouraged to ensure step down was carried out for new staff before laying off old staff. Strict supervision by facility head and supervisors was encouraged. All of this is to ensure that facilities optimally avoid rejection, and this will result in the early generation of EID result (shortened TAT) and will help in better clinical judgement by the clinicians, reduce mortality and morbidity rate in EID with a subsequent increase in Prevention of Mother to Child Transmission.

\section{RESULtS}

\section{A. Results of Elements Measured at Baseline}

Fig. 1 shows average percentage rate of EID rejection, within a baseline period of seven months. During the baseline period, sample rejection rate started at a rate of $0 \%$ in January increased to 5\% in February and went back to $0 \%$ in March. In April however, the rate of rejection increased to 9\%. There was a decline in rejection rate to $5 \%$ and $7 \%$ in May and June. A sudden spike in rejection occurred in July. Root cause analysis was carried out to know reasons for the high rate of EID rejections. It was found that facilities with the higher rates of rejection were private Clinics who lay off their staff and do not border to do step down for the newly employed; staff not acquainted with the national algorithm for acceptance and rejection of EID samples. Most staff, who work with these private facilities are poorly remunerated, hence lack dedication to duties and competence.

\section{B. Result of Elements Measured at Final Period}

The final data showed a tremendous decrease in EID rejection rate from the months of January to March 2019, at $1 \%-0 \%$ rate respectively, after interventions have been put in place. It shows that improvements have been made and this has resulted in lower TAT, few, or no samples rejection. This will impact positively on the exposed infants as the positive ones will be enrolled immediately in ART clinic. Shortened TAT helps in quick diagnosis and treatment with an overall satisfaction of patients. Before the commencement of this study, EID TAT was longer than one month. Results of a batch of samples sent from a particular facility will be sent back to same facility alongside rejected forms. Hence, exposed HIV infants whose samples were rejected have to wait until results are sent back to the facility before recollection of samples can be made in about seven weeks. This impacted negatively on the exposed HIV infants since proper clinical decisions could not be made. The study however, brought about a reduction in TAT to as low as 1-2 weeks, with a minimal rate of sample rejection at the point of sample receipt.

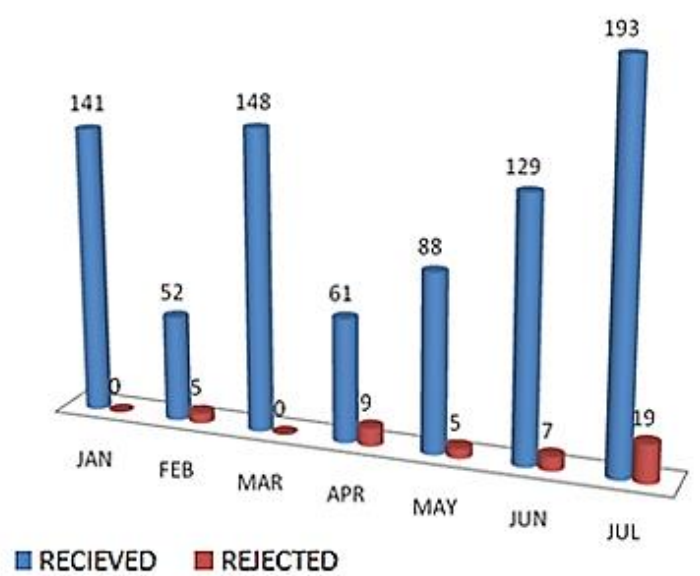

Fig. 1. Percentage rate of EID samples received and rejected from January-July 2019.

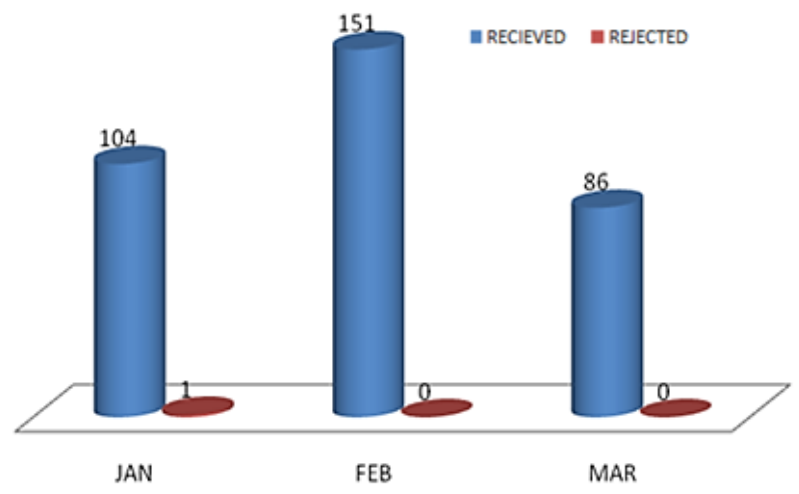

Fig. 2. Percentage rate of EID samples received and rejected from January - March 2020.

\section{CONCLUSION}

The aim of this project was to reduce EID rejection rate to $1 \%$. Findings have however, demonstrated a significant dropin rejection rate as compared to baseline and final results. It shows that EID sample rejection can actually be eradicated if all interventional factors used in this study are applied.

The challenges encountered in this project include the following: Difficulty in getting the sending facility adhere to change; Incessant transfer/dropping of staff by private facility management; Logistics.

The following recommendations were proffered: Proper step down to new staff before quitting old staff; Competency assessment of new staff; Strict and constant monitoring by supervisors; Adherence to rejection/acceptance criteria.

\section{REFERENCES}

[1] G. J. Fermann and J. Suyama, "Point of care testing in the emergency department". Journal of Emergency Medicine, vol. 22, pp. 393-404, 2002. 
[2] B. Bergman and B. Klefsjo, Quality: From customer needs to customer satisfaction. Maidenhead, England: McGraw-Hill, 1994.

[3] N. B. Watts, "Reproducibility (precision) in alternate site testing. A clinician's perspective." Archives of Pathology \& Laboratory Medicine, vol. 119, pp. 914-917, 1995.

[4] C. R. Handorf, "College of American Pathologists Conference XXVIII on alternate site testing: Introduction." Archives of Pathology \& Laboratory Medicine, vol. 119, pp. 867-73, 1995.

[5] P. J. Howanitz, and S. J. Steindel, "Intra-laboratory performance and laboratorians' expectations for stat turnaround times. A College of American Pathologists Q-Probes study of four cerebrospinal fluid determinations". Archives of Pathology \& Laboratory Medicine, vol. 115, pp. 977-983, 1991.

[6] P. A. Wayne, Application of a Quality Management System Model for Laboratory Services, Clinical and Laboratory Standards Institute. 3rd ed. CLSI; Document GP26-A3, pp. 1887-1898, 2004.

[7] The Joint Commission, National patient safety goals: laboratory services program, 2009.

[8] World Health Organization, Antiretroviral drugs for treating pregnant women and preventing HIV infection in infants-recommendations for a public health approach: 2010 version," WHO, Geneva. Available: http://whqlibdoc.who.int/publications/2010/9789241599818_eng.pdf

[9] J. A. Volmink, and B. J. Marais, HIV: Mother-to-Child Transmission,' Clinical Evidence (Online), 2008, Published Online 5 February. Available: http://www.ncbi.nlm.nih.gov/pmc/articles/PMC2907958/

[10] M. L. H. Newell, M. Coovadia, N. Cortina-Borja, P. Rollins, S Gaillard, and F. Dabis, "Ghent International AIDS Society (IAS) Working Group on HIV Infection in Women and Children, Mortality of Infected and Uninfected Infants Born to HIV-Infected Mothers in Africa: A Pooled Analysis.” Lancet, vol. 364, no. 9441, pp. 1236- 1243, 2004.

[11] K. Mcintosh, J. Pitt, and D. Brambilla, "Blood culture in the first 6 months of life for the diagnosis of vertically transmitted human immunodeficiency virus infection: The women and infants transmission study group." The Journal of Infectious Diseases, vol. 17, no. 4, pp. 996-1000, 1994.

[12] G. G. Sherman, P. A. Cooper, A. H. Coovadia, A. J. Puren, S. A. Jones, and M. Mokhachane, "Polymerase chain reaction for diagnosis of human immunodeficiency virus infection in infancy in low resource settings." Paediatric Infectious Disease Journal, vol. 24, no. 11, pp. 993-997, 2005.

[13] T. L. Creek, G. G. Sherman, J. Lu, L. T. Finkbeiner, and M. G. Fowler, "Infant human immunodeficiency virus diagnosis in resource-limited settings: Issues, technologies, and country experiences." American Journal of Obstetrics \& Gynecology, vol. 197, no. 3, S64-S71, 2007.

[14] W. Stevens, G. Sherman, R. L. Downing, M. C. Parsons, and Y. S. Crowley, "Role of the Laboratory in Ensuring Global Access to ARV Treatment for HIV-Infected Children: Consensus Statement on the Performance of Laboratory Assays for Early Infant Diagnosis." Open Chemical Physics Journal, vol. 2, pp. 17-25, 2008.

[15] J. S. Read, "Diagnosis of HIV-1 infection in children younger than 18 months in the United States". Pediatrics, vol. 120, no. 6, e1547-e1562, 2007.

[16] M. Tamhane, B. Gautney, and C. Shiu, "Analysis of the optimal cutpoint for HIV-p24 antigen testing to diagnose HIV infection in HIV exposed children from resource-constrained settings." Journal of Clinical Virology, vol. 50, no. 4, pp. 338-341, 2011.

[17] M. J. Wessman, Z. T. Heilgaard, and T. L. Katzenstein, "Determination of HIV status of infants born to HIV-infected mothers: A review of the diagnostic methods with special focus on the applicability of p24 antigen testing in developing countries." Infectious Diseases, vol. 44 no. 3, pp. 209-215, 2012.

[18] American Academy of Pediatrics Committee on Pediatric AIDS, "HIV testing and prophylaxis to prevent mother-to-child transmission in the United States." Paediatrics, vol. 122, no. 5, pp. 1127-1134, 2010.

[19] R. J. S. Kerr, G. S. Player, A. Fiscus and J. A. E. Nelson, "Qualitative human immunodeficiency virus RNA analysis of dried blood spots for diagnosis of infections in infants." Journal of Clinical Microbiology, vol. 47, no. 1, pp. 220-222, 2009.

[20] M. Hamufare, M. Owen, C. Addmore, "Early Infant Diagnosis Sample Management in Mashonaland West Province Zimbabwe." Public Library of Science, vol. 9, no. 3, p. 61, 2017.

[21] N. Grüner, O. Stambouli, R. S. Ross, "Dried blood spots - preparing and processing for use in immunoassays and in molecular techniques. Journal of Visualized Experiments," in A Treatise on Electricity and Magnetism, C. Maxwell, ed. 3rd ed., vol. 2. Oxford: Clarendon, 1892, pp.68-73, 2015. 